\title{
Influence of the anterior arch shape and root position on root angulation in the maxillary esthetic area
}

\author{
Suweera Petaibunlue(D) ${ }^{1}$, Pravej Serichetaphongse $\mathbb{D}^{1,2}$, Atiphan Pimkhaokham $\mathbb{D}^{1,3, *}$ \\ ${ }^{1}$ Esthetic Restorative and Implant Dentistry Program, Faculty of Dentistry, Chulalongkorn University, Bangkok, Thailand \\ ${ }^{2}$ Department of Prosthodontics, Faculty of Dentistry, Chulalongkorn University, Bangkok, Thailand \\ ${ }^{3}$ Department of Oral and Maxillofacial Surgery, Faculty of Dentistry, Chulalongkorn University, Bangkok, Thailand
}

\section{ABSTRACT}

Purpose: This study was conducted to characterize the relationship of the angulation between the tooth root axis and alveolar bone axis with anterior alveolar (AA) arch forms and sagittal root position (SRP) in the anterior esthetic region using cone-beam computed tomography (CBCT) images.

Materials and Methods: CBCT images that met the inclusion and exclusion criteria were categorized using a recent classification of AA arch forms and a SRP classification. Then, the angulation of the root axis and the alveolar bone axis was measured using mid-sagittal CBCT images of each tooth. The relationships of the angulation with each AA arch form and SRP classification were evaluated using 1-way analysis of variance and a linear regression model.

Results: Ninety-eight CBCT images were included in this study. SRP had a greater influence than the AA arch form on the angulation of the root axis and the alveolar bone axis $(P<0.05)$. However, the combination of AA arch form and SRP was more predictive of the angulation of the root axis and the alveolar bone axis than either parameter individually.

Conclusion: The angulation of the root axis and alveolar bone axis demonstrated a relationship with the AA arch form and SRP in teeth in the anterior esthetic region. The influence of SRP was greater, but the combination of both parameters was more predictive of root-to-bone angulation than either parameter individually, implying that clinicians should account for both the AA arch form and SRP when planning implant placement procedures in this region.

(Imaging Sci Dent 2019; 49: 123-30)

KEY WORDS: Alveolar Process; Cone-Beam Computed Tomography; Tooth Root; Maxilla

\section{Introduction}

Dental implants have become the standard treatment for dental reconstruction due to their high survival and success rates for both osseointegration and restoration. However, dental implants in the maxillary anterior esthetic zone are challenging for clinicians due to patients' esthetic expectations and several risk factors that affect treatment outcomes, including anatomical risks, aspects of the smile design, and limited tooth space. ${ }^{1-5}$

Received January 24, 2019; Revised March 23, 2019; Accepted April 10, 2019 *Correspondence to : Prof. Atiphan Pimkhaokham

Department of Oral and Maxillofacial Surgery, Faculty of Dentistry, Chulalongkorn University, 34 Henri-Dunant Road, Wangmai, Pathumwan, Bangkok 10330, Thailand Tel) 66-2-2188581,E-mail) atiphan.p@chula.ac.th
From a biomechanical perspective, the anterior maxilla is the weakest region for implant placement, which underscores the importance of placing implants with an appropriate 3-dimensional position and angulation in the alveolar arch to optimize outcomes related to esthetics, phonetics, load distribution, and the loss of bone and soft tissue around the implant. ${ }^{1}$ After tooth extraction, hard and soft tissue alterations can occur in the both horizontal and vertical dimensions, especially on the facial aspect of the alveolar ridge ${ }^{6,7}$ When a large amount of alveolar bone change occurs, there may be insufficient ridge width for implant placement. ${ }^{4}$ A clinical examination and proper radiographic images, such as those provided by 3 -dimensional computed tomography, are required to obtain accurate information

Copyright (c) 2019 by Korean Academy of Oral and Maxillofacial Radiology

This is an Open Access article distributed under the terms of the Creative Commons Attribution Non-Commercial License (http://creativecommons.org/licenses/by-nc/3.0) which permits unrestricted non-commercial use, distribution, and reproduction in any medium, provided the original work is properly cited. Imaging Science in Dentistry · pISSN 2233-7822 eISSN 2233-7830 
about the alveolar arch prior to implant placement.

Several reports have presented classifications of the maxillary arch form or the dental arch form using measurements derived from models ${ }^{8-10}$ or human cadavers. ${ }^{11}$ However, previous reports have all focused on the dental arch or basal arch, and none have fully addressed the form of the alveolar arch proper, since the alveolar process that supports teeth and dental implants is located deep under the tooth structure. Suk et al. (2013) were the first to report the application of cone-beam computed tomography (CBCT) to compare dental and basal arch forms in cases of normal occlusion and class III malocclusion. ${ }^{12}$ Using CBCT, Bulyalert et al. (2018) recently reported a classification of alveolar arch forms in the maxillary anterior esthetic zone. ${ }^{13}$

Anterior alveolar (AA) arch forms can be classified into 4 groups: long narrow arches, short medium arches, long medium arches, and long wide arches, according to the intercanine width, interpremolar width, intercanine depth, and intercanine width-to-depth ratio. ${ }^{13}$ This classification of AA arch forms could be helpful for selecting the implant size when determining the number of implants or implant axes; however, it still has no evidential support.

The root position is crucial for implant treatment planning in the anterior esthetic region, particularly in immediate implant therapy. The original root position in the alveolar bone was found to explain the morphology of the post-extraction site, which in turn could predict future implant stability and bone perforation. Accordingly, Kan et al. ${ }^{14}$ presented a classification of sagittal root position (SRP) to aid implant treatment planning, in which the relationship between the root position and its osseous housing is categorized as class I, II, III, or IV.

Several studies have reported the angulation of the alveolar bone axis and the long axis of the whole tooth for the anterior maxillary teeth. This information is beneficial for guiding orthodontic therapies. ${ }^{15-17}$ Nonetheless, some authors have used the SRP classification within the alveolar bone housing ${ }^{14}$ to evaluate bone quality as a guideline for assessing the challenges of implant placement or the need for adjunctive soft tissue and/or hard tissue augmentation during treatment planning. ${ }^{18-20}$ Wang et al. ${ }^{15}$ and Lau et al. ${ }^{21}$ suggested that the implant axis should be placed in a way that mimics the natural tooth root and is parallel to the tooth root axis. Nevertheless, no reports have yet demonstrated the proper angulation of the natural tooth root and the alveolar bone axis.

Taken together, achieving long-term successful outcomes of implant therapy requires the implant fixture to be placed in the alveolus with an appropriate position and angulation ${ }^{1}$ depending on various factors, such as the arch form. Nonetheless, no previous study has reported the angulation of the root axis and the alveolar bone axis in different types of anterior maxillary alveolar arch forms. This study therefore aimed to characterize the relationships of the angulation between the tooth root axis and the alveolar bone axis with AA arch forms and SRP in the anterior esthetic region using CBCT images.

\section{Materials and Methods}

This study was conducted with the approval of the Ethics Committee of the Faculty of Dentistry, Chulalongkorn University, Bangkok, Thailand (HREC-DCU-P 2016-011). CBCT images of the anterior maxillary region, including the right to the left maxillary first premolars of patients without any artifacts and defects, along with patients' data, were retrieved from the radiographic database of the Esthetics and Implant Clinic at Chulalongkorn University. The data were originally recorded from January 2013 to December 2016. The following inclusion criteria were applied: age of at least 21 years at the time of the CBCT scan; class I normal occlusion (a class I molar and canine relationship, a curve of Spee of less than $2 \mathrm{~mm}$, arch length discrepancies of less than $3 \mathrm{~mm}$, and normal overbite and overjet); fully formed and intact maxillary incisors, canines and first premolars; no evidence of periapical pathology; and no history of surgical and/or orthodontic treatment. The measurements were made using computer software (One Volume Viewer Software Ver.1.8.0; J. Morita Mfg. Corp., Kyoto, Japan) under 300\% magnification.

\section{Classification and measurement of the $A A$ arch form}

In this study, the AA alveolar arch form was classified according to the system proposed by Bulyalert et al. ${ }^{13}$ Briefly, the classification was based on the curve of the anterior maxillary alveolar arch from the right to left maxillary first premolar teeth at the implant platform level, approximately $3 \mathrm{~mm}$ below the cemento-enamel junction. The anterior maxillary alveolar arch forms were classified into 4 groups: long narrow arch forms, short medium arch forms, long medium arch forms, and long wide arch forms. ${ }^{13}$

\section{SRP classification}

The SRP classification was based on the dental root position of the maxillary incisors and maxillary first premolars in the alveolar bone housing. Each tooth root image was classified according to the classification published 


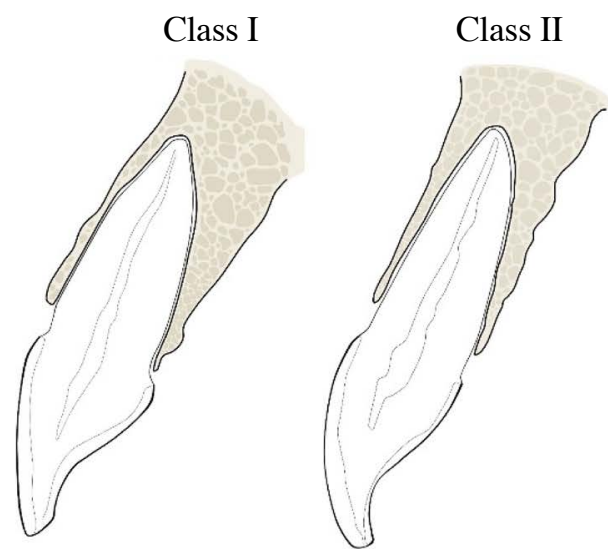

Class III

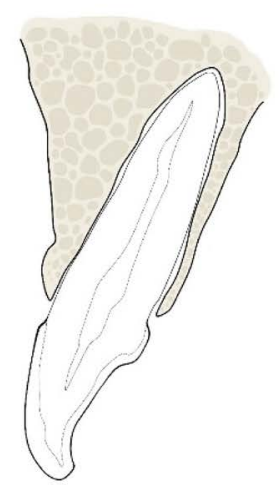

\section{Class IV}

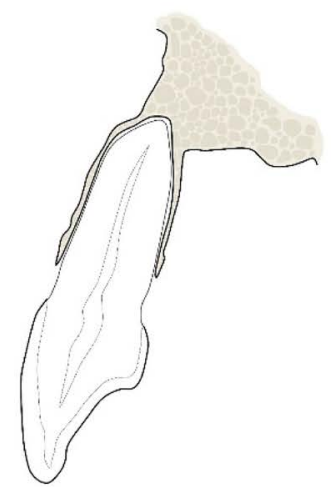

Fig. 1. The sagittal root position classification as reported by Kan et al. ${ }^{14}$
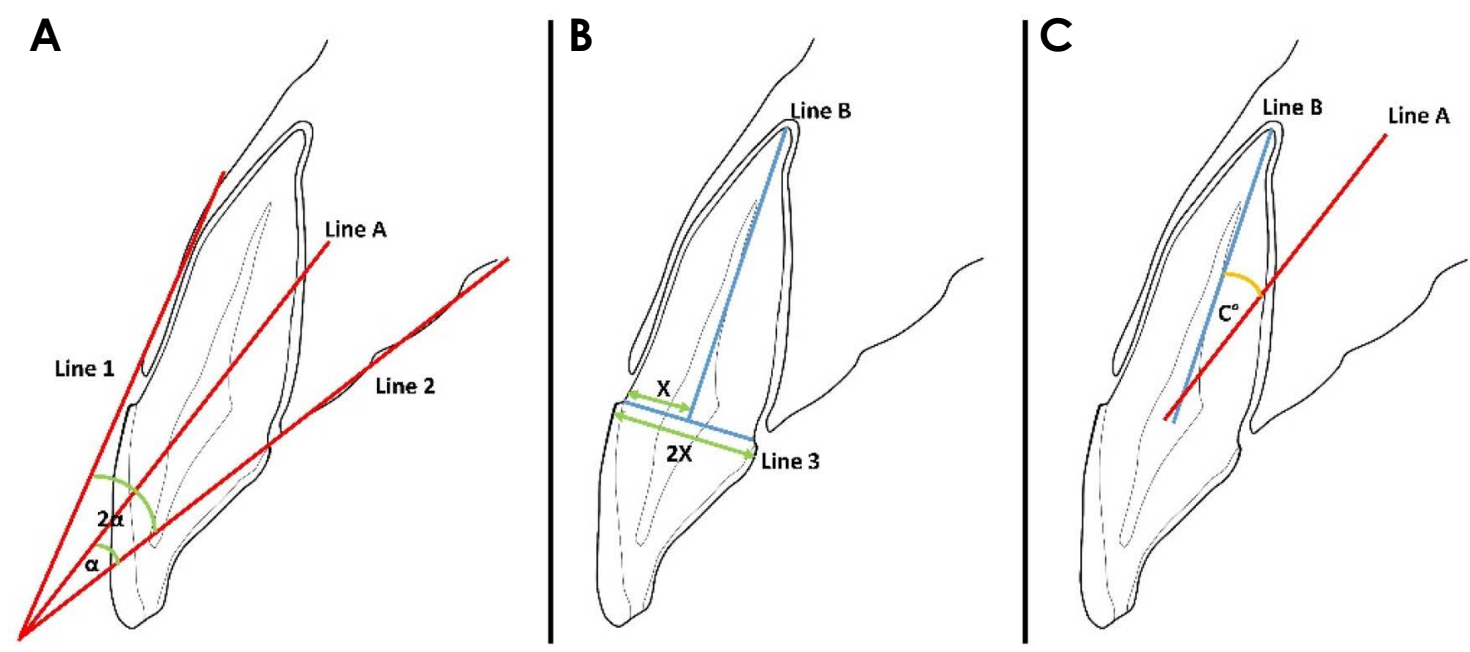

Fig. 2. The landmarks for angulation evaluation comprise the alveolar bone axis, tooth root axis, and the angle between the alveolar bone axis and the tooth root axis. A. Line A: alveolar bone axis, line 1: the buccal line; and line 2: the palatal line. B. Line B: the tooth root axis; line 3: the cervical line. C. $\mathrm{C}^{\circ}$ is defined as the angle between the alveolar bone axis (line A) and the tooth root axis (line B).

by Kan et al., ${ }^{14}$ according to which the position of a root within its bone is divided into 4 classes,${ }^{14}$ as detailed in Figure 1.

As shown in Figure 1, class I contains roots engaged with the buccal cortical bone, class II includes roots that are in the middle of the alveolar bone housing without engaging either the buccal or the palatal bone at the apical third of the root, class III contains roots engaged with the palatal cortical bone, and class IV refers to roots engaged with both the buccal and the palatal cortical bone. ${ }^{14}$

\section{Angulation evaluation}

The angulation of the alveolar bone axis and the tooth root axis was defined as the angle between the alveolar bone axis and the tooth root axis of the maxillary central incisors, lateral incisors, canines, and first premolar teeth. To measure the angulation of the alveolar bone axis and the tooth root axis, the labio-lingual cross-section at the middle of the tooth was measured using CBCT images in accordance with the procedure described by Lau et al. ${ }^{21}$ Figure 2 shows the angulation and long axis of the tooth root and alveolar bone. Specifically, Figure 2A shows the alveolar bone axis, which is represented by line A, which bisects the angle of the buccal line (line 1) and palatal line (line 2). Figure 2B demonstrates the tooth root axis (line $\mathrm{B})$, which is the line drawn from the midpoint of the cervical line (line 3) to the root apex. The angulation between the alveolar bone axis and the tooth root axis is shown in Figure $2 \mathrm{C}$, where $\mathrm{C}^{\circ}$ represents the angle between the alveolar bone axis (line A) and the tooth root axis (line B). 
The angulation between the alveolar bone axis and the root axis was analyzed in both the right and left maxillary central incisors, the maxillary lateral incisors, the maxillary canines, and the maxillary first premolars.

Table 1. Sagittal angles between the root axis and alveolar bone axis (unit: degrees)

\begin{tabular}{lcr}
\hline \multicolumn{1}{c}{ Tooth } & $\begin{array}{c}\text { Sagittal angle between root axis } \\
\text { and alveolar bone axis }\end{array}$ & Range \\
\hline $\begin{array}{l}\text { Maxillary central } \\
\text { incisor }(\mathrm{n}=196)\end{array}$ & $16.59 \pm 5.97^{\mathrm{A}}$ & $1.10-33.12$ \\
$\begin{array}{l}\text { Maxillary lateral } \\
\text { incisor (n=196) }\end{array}$ & $13.89 \pm 6.12^{\mathrm{B}}$ & $0.67-32.41$ \\
$\begin{array}{l}\text { Maxillary canine } \\
(\mathrm{n}=196)\end{array}$ & $14.93 \pm 6.02^{\mathrm{B}}$ & $-0.61-35.23$ \\
$\begin{array}{l}\text { Maxillary first } \\
\text { premolar ( } \mathrm{n}=196)\end{array}$ & $13.38 \pm 6.46^{\mathrm{B}}$ & $1.01-30.64$ \\
\hline
\end{tabular}

The same superscript capital letters indicate the absence of significant differences in sagittal angulation $(P>0.05)$.

\section{Data analysis}

The mean and standard deviation of the angulation between the tooth root axis and the alveolar bone axis was calculated for each of the different types of AA arch forms. The data were analyzed using statistical software (SPSS 22.0; IBM Corp., Armonk, NY, USA). To ensure the reliability of the measurements obtained from the examiner, intra-examiner calibration was performed by measuring 10 randomly selected images twice on separate days 1 month after the initial measurements. The intraclass correlation coefficient was calculated using a 2-way mixed effects model to obtain a $95 \%$ level of confidence interval. The normality of the data was determined using the Shapiro-Wilk test. A comparative analysis with the independent t-test and Pearson product moment correlation was conducted between the right and left sides of the alveolar arch forms. Descriptive statistics were presented, including mean values with standard deviations, frequencies, and percentages. Analysis of variance with the Scheffe post-hoc test was performed

Table 2. Comparison of mean values and standard deviations of the sagittal angle of the root axis and alveolar bone axis across the 4 groups defined by anterior alveolar arch form

\begin{tabular}{lllll}
\hline $\begin{array}{c}\text { Arch form } \\
\text { tooth }\end{array}$ & \multicolumn{1}{c}{$\begin{array}{c}\text { Long narrow } \\
(\mathrm{n}=60)\end{array}$} & $\begin{array}{c}\text { Short medium } \\
(\mathrm{n}=24)\end{array}$ & $\begin{array}{c}\text { Long medium } \\
(\mathrm{n}=60)\end{array}$ & $\begin{array}{c}\text { Long wide } \\
(\mathrm{n}=52)\end{array}$ \\
\hline Central incisor & $15.34 \pm 5.88^{\mathrm{A}, \mathrm{B}, \mathrm{C}, \mathrm{D}}$ & $13.49 \pm 4.93^{\mathrm{A}}$ & $18.01 \pm 5.19^{\mathrm{B}, \mathrm{D}}$ & $17.81 \pm 6.64^{\mathrm{C}, \mathrm{D}}$ \\
Lateral incisor & $13.40 \pm 6.35$ & $11.24 \pm 6.62$ & $14.78 \pm 5.75$ & $14.66 \pm 5.79$ \\
Canine & $15.06 \pm 6.79^{\mathrm{A}, \mathrm{B}, \mathrm{C}}$ & $11.57 \pm 4.42^{\mathrm{B}}$ & $16.10 \pm 5.99^{\mathrm{C}}$ & $14.99 \pm 5.29^{\mathrm{A}, \mathrm{B}, \mathrm{C}}$ \\
First premolar & $12.35 \pm 5.97$ & $13.60 \pm 6.28$ & $13.05 \pm 6.46$ & $14.86 \pm 6.98$ \\
\hline Overall & $14.04 \pm 6.34^{\mathrm{A}, \mathrm{B}, \mathrm{C}, \mathrm{D}}$ & $12.48 \pm 5.65^{\mathrm{A}}$ & $15.09 \pm 6.37^{\mathrm{B}, \mathrm{D}}$ & $15.58 \pm 6.30^{\mathrm{C}, \mathrm{D}}$ \\
\hline
\end{tabular}

The same superscript capital letters indicate the absence of significant differences in sagittal angulation for each horizontal row $(P>0.05)$.

Table 3. Distribution of sagittal root positions of the anterior maxillary incisors and first premolars in the alveolar bone

\begin{tabular}{lccccc}
\hline Sagittal root position & Overall & Central incisor & Lateral incisor & Canine & First premolar \\
\hline Class I & $667(85.10 \%)$ & $156(79.60 \%)$ & $160(84.20 \%)$ & $194(99.00 \%)$ & $152(77.60 \%)$ \\
Class II & $82(10.50 \%)$ & $40(20.40 \%)$ & $10(5.10 \%)$ & $1(0.50 \%)$ & $31(15.80 \%)$ \\
Class III & - & - & - & - & $1(0.50 \%)$ \\
Class IV & $35(4.50 \%)$ & - & $19(10.70 \%)$ & $13(6.60 \%)$ \\
\hline Total & 784 & 196 & 196 & 196 \\
\hline
\end{tabular}

Table 4. Angulation of the maxillary incisors and first premolars with reference to the alveolus according to the sagittal root position (SRP) classification $^{14}$

\begin{tabular}{lcccc}
\hline \multicolumn{1}{c}{ SRP tooth } & Class I & Class II & Class III & Class IV \\
\hline Central incisor & $17.27 \pm 5.60^{\mathrm{A}}$ & $13.91 \pm 6.66$ & - & - \\
Lateral incisor & $15.11 \pm 5.62^{\mathrm{B}, \mathrm{C}}$ & $11.42 \pm 4.62$ & - & $5.49 \pm 2.69$ \\
Canine & $14.99 \pm 6.02^{\mathrm{B}, \mathrm{D}}$ & $8.63 \pm 0.00$ & - & $9.84 \pm 0.00$ \\
First premolar & $14.09 \pm 6.14^{\mathrm{B}, \mathrm{E}}$ & $12.43 \pm 7.61$ & - & $7.37 \pm 3.26$ \\
Overall & $15.35 \pm 5.96^{*}$ & $12.98 \pm 6.80^{\S}$ & - & $6.31 \pm 3.04^{\dagger}$ \\
\hline
\end{tabular}

The same superscript capital letters indicate the absence of significant differences in angulation for each column $(P>0.05)$. The same symbols indicate the absence of significant differences in angulation for each horizontal row $(P>0.05)$. 
to compare the angulation of the root axis and the alveolar bone axis of the maxillary incisors and maxillary first premolars according to the type of alveolar arch form and the SRP. The Tukey post-hoc test was used to compare the sagittal angulation of individual teeth. The influence of the AA arch form and SRP on the angulation between the root axis and the alveolar bone axis was analyzed through linear regression models. $P$ values $<0.05$ were considered to indicate statistically significant differences.

\section{Results}

In total, CBCT images from 98 patients satisfied the inclusion criteria of this study, and 196 CBCT images of the left and right maxillary central incisors, lateral incisors, canines, and first premolars were evaluated. The mean sagittal angles between the root axis and the respective alveolar bone axis of each tooth are shown in Table 1 . The largest sagittal angulation between the alveolar bone axis and the tooth root axis was found for the maxillary central incisor. There was no significant difference in the sagittal angulation of the alveolar bone axis and the tooth root axis between the right and left sides. However, a moderate correlation was found between the right and the left $\operatorname{sides}(\mathrm{r}=0.671 ; P<0.001)$.

The classification of the AA arch forms showed that in the CBCT images, there were 30 long narrow arches, 12 short medium arches, 30 long medium arches, and 26 long wide arches. The overall mean sagittal angulation of the root axis and alveolar bone axis in the short medium arches was significantly lower than that observed in both the long medium arches and the long wide arches. The sagittal angulation between the alveolar bone axis and root axis in the short medium arches showed less significant differences than were observed in the long medium arch at the maxillary central incisor and canine. In addition, the sagittal angulation between the alveolar bone axis and root axis of the maxillary central incisor of long wide arches was significantly greater than was observed for short medium arches (Table 2).

The SRP was categorized according to Kan et al. ${ }^{14}$ Most of the roots of maxillary incisors and maxillary first premolars were positioned buccally within the alveolar bone (class I). Meanwhile, no cases were found in which the SRP showed engagement with the palatal cortical bone (class III). The number (percentage) of teeth showing class I, II, III, and IV SRP is shown in Table 3. Statistically significant differences in the overall mean angles between the tooth root axis and the alveolar bone axis were

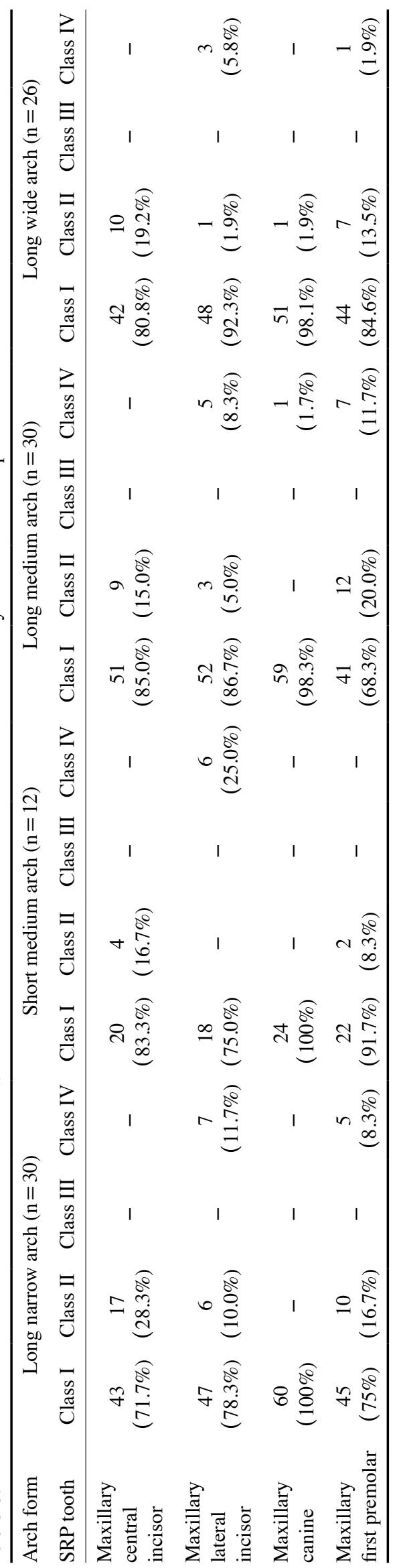


Table 6. Linear regression analysis of the anterior alveolar (AA) arch form and sagittal root position (SRP) in relation to the sagittal root angulation between the tooth root axis and the alveolar bone axisa

\begin{tabular}{|c|c|c|c|c|c|c|}
\hline & \multicolumn{2}{|c|}{ AA arch form } & \multicolumn{2}{|c|}{ SRP } & \multicolumn{2}{|c|}{ AA arch form* SRP } \\
\hline & $\beta$ & $P$ value & $\beta$ & $P$ value & $\beta$ & $P$ value \\
\hline (Constant) & 15.579 & $<0.001$ & 6.314 & $<0.001$ & 7.101 & $<0.001$ \\
\hline Long narrow & -1.544 & 0.009 & & & -1.161 & 0.038 \\
\hline Short medium & -3.102 & $<0.001$ & & & -2.787 & $<0.001$ \\
\hline Long medium & -0.091 & 0.876 & & & 0.239 & 0.669 \\
\hline SRP class I & & & 9.036 & $<0.001$ & 8.867 & $<0.001$ \\
\hline SRP class II & & & 6.668 & $<0.001$ & 6.482 & $<0.001$ \\
\hline SRP class III & & & - & - & - & - \\
\hline $\mathrm{R}$ & 0.170 & & 0.312 & & 0.350 & \\
\hline Adjusted R-square & 0.025 & & 0.095 & & 0.117 & \\
\hline F-value & 7.777 & & 42.138 & & 21.773 & \\
\hline$P$-value & $<0.001$ & & $<0.001$ & & $<0.001$ & \\
\hline
\end{tabular}

*Dependent variable: angle

found between classes I, II, and IV $(P<0.05)$. In class I, the mean angulation of the maxillary central incisor was significantly greater than that of the other teeth. However, in classes II and IV, significant differences between the mean root-to-bone angulation of the maxillary incisors and the first premolar were not found (Table 4).

The frequency of each SRP class according to each AA arch form for the various teeth is shown in Table 5. The SRP of the maxillary central incisor in every arch form was either class I or II. More than $98 \%$ of the maxillary canines were classified as SRP class I, as were most of the maxillary first premolars, followed by classes II and IV, respectively.

As shown in Table 6, the alveolar arch form and SRP classification explained $2.5 \%$ and $9.5 \%$ of the variation in the angle of the root axis and the alveolar bone axis, respectively. However, the AA arch form in combination with the SRP accounted for approximately $11.7 \%$ of the variation in the angulation of the root axis and alveolar bone axis. The relationship between the alveolar arch form and the SRP with the angle of the root axis and the alveolar bone axis was found to follow the following equation:

Angle $=7.101-1.161$ long narrow arch -2.787 short medium arch +0.239 long medium arch +8.867 $\mathrm{SRP}_{1}+6.482 \mathrm{SRP}_{2}$

\section{Discussion}

Our study showed a moderate correlation between the right and left AA arch forms. The angulation between the dental root axis and the alveolar bone axis was also correlated to a similar extent. Thereby, the position and axis of an implant in the anterior maxillary region could be guided by the angulation of the contralateral tooth root axis and the alveolar bone axis. Adjunctive bone augmentation may be required to build an appropriate contour of the AA arch. ${ }^{22,23}$

Our analysis of the relationship between the root-tobone angulation and the AA arch form demonstrated that the angulation between the tooth root axis and the alveolar bone axis was strongly influenced by the intercanine depth. The angulation of the tooth root axis and alveolar bone axis decreased as the intercanine depth of the alveolar arch became smaller. Thus, the type of AA arch form could be used to predict the angulation of the tooth root axis and the alveolar bone axis.

Class I SRP showed the greatest angulation between the dental root axis and the alveolar bone axis, and exhibited greater palatal bone thickness than the other classes. ${ }^{24}$ Most of the maxillary teeth in the anterior esthetic zone in this study were classified as having class I SRP, whereas class III SRP was not found within the dataset used. This result is consistent with the results of previous studies. The frequency of class III SRP has been reported to vary from $0.2 \%$ to $1.8 \%,{ }^{14,21,25-27}$ indicating the rarity of this class of SRP. ${ }^{14,21,25-27}$ Therefore, palatal implant engagement in the anterior maxilla is recommended due to the availability of sufficient palatal bone support, which affects primary implant stability during immediate implant placement. Consequently, immediate implant placement is typically performed in the anterior esthetic region.

The previous concept of immediate implant placement was driven by restoration, ${ }^{28,29}$ meaning that the implant should be placed in a way that mimics the dental root axis. Since class I SRP is most common, most implants should 
be drilled parallel to the labial cortical bone, and should also be inclined more towards the labial edge than towards the incisal edge or cingulum. For this reason, the thinned alveolar bone, especially in the labial aspect, tended to increase the risk of bone perforation during osteotomy. On the basis of the findings of this study, we recommend the use of CBCT assessment as a standard method for presurgical evaluation of the implant site to determine the volume and quality of the bone surrounding vital structures. In order to minimize recession of the labial bone and soft tissue, we recommend a minimum labial bone thickness of 1-2 mm. ${ }^{30,31}$ As a result, when seeking to achieve long-term maintenance of both esthetic results and function, surgeons should use a modified 3-dimensional implant position and angulation by placing a properly sized and shaped implant fixture more palatally, and should also fill the labial gap with bone grafting material during immediate implant placement. Moreover, if the bone volume is insufficient, bone augmentation may be performed either during or before implant placement. ${ }^{32,33}$

In summary, the angulation of the dental root axis and the alveolar bone axis plays an important role in determining implant position, and is influenced by both the AA arch form and SRP. Surgeons should be aware of this information and take it into consideration when determining where implants should be properly placed in order to achieve a suitable result.

\section{Acknowledgements}

We thank Associate Professor Pagaporn Pantuwadee Pisarnturakit for assistance with the statistical analysis.

\section{References}

1. Buser D, Martin W, Belser UC. Optimizing esthetics for implant restorations in the anterior maxilla: anatomic and surgical considerations. Int J Oral Maxillofac Implants 2004; 19 Suppl: 43-61.

2. Januário AL, Duarte WR, Barriviera M, Mesti JC, Araújo MG, Lindhe J. Dimension of the facial bone wall in the anterior maxilla: a cone-beam computed tomography study. Clin Oral Implants Res 2011; 22: 1168-71.

3. Johnson K. A study of the dimensional changes occurring in the maxilla following tooth extraction. Aust Dent J 1969; 14: 241-4.

4. Misch CE. Anterior single-tooth replacement: surgical considerations. In: Misch CE. Contemporary implant dentistry. 3rd ed. Canada: Mosby Elsevier; 2008. p. 739-68.

5. Moy PK, Pozzi A, Beumer III J. Surgical Considerations for the Esthetic zone. In: Beumer J, Faulkner RF, Shah K, Moy
PK. Fundamentals of implant dentistry: surgical principles. Hanover Park, IL: Quintessence Publishing; 2016. p. 291-332.

6. Jahangiri L, Devlin H, Ting K, Nishimura I. Current perspectives in residual ridge remodeling and its clinical implications: a review. J Prosthet Dent 1998; 80: 224-37.

7. Van der Weijden F, Dell'Acqua F, Slot DE. Alveolar bone dimensional changes of post-extraction sockets in humans: a systematic review. J Clin Periodontol 2009; 36: 1048-58.

8. Ferrario VF, Sforza C, Miani A Jr, Tartaglia G. Mathematical definition of the shape of dental arches in human permanent healthy dentitions. Eur J Orthod 1994; 16: 287-94.

9. Preti G, Pera P, Bassi F. Prediction of the shape and size of the maxillary anterior arch in edentulous patients. J Oral Rehabil 1986; 13: 115-25.

10. Sagat G, Yalcin S, Gultekin BA, Mijiritsky E. Influence of arch shape and implant position on stress distribution around implants supporting fixed full-arch prosthesis in edentulous maxilla. Implant Dent 2010; 19: 498-508.

11. Pietrokovski J, Starinsky R, Arensburg B, Kaffe I. Morphologic characteristics of bony edentulous jaws. J Prosthodont 2007; 16: 141-7.

12. Suk KE, Park JH, Bayome M, Nam YO, Sameshima GT, Kook YA. Comparison between dental and basal arch forms in normal occlusion and Class III malocclusions utilizing conebeam computed tomography. Korean J Orthod 2013; 43: 1522.

13. Bulyalert A, Pimkhaokham A. A novel classification of anterior alveolar arch forms and alveolar bone thickness: a cone beam computed tomography study. Imaging Sci Dent 2018; 48: 191-9.

14. Kan JY, Roe P, Rungcharassaeng K, Patel RD, Waki T, Lozada JL, et al. Classification of sagittal root position in relation to the anterior maxillary osseous housing for immediate implant placement: a cone beam computed tomography study. Int J Oral Maxillofac Implants 2011; 26: 873-6.

15. Wang HM, Shen JW, Yu MF, Chen XY, Jiang QH, He FM. Analysis of facial bone wall dimensions and sagittal root position in the maxillary esthetic zone: a retrospective study using cone beam computed tomography. Int J Oral Maxillofac Implants 2014; 29: 1123-9.

16. Zhang S, Shi X, Liu H. Angulations of anterior teeth with reference to the alveolar bone measured by CBCT in a Chinese population. Implant Dent 2015; 24: 397-401.

17. Masunaga M, Ueda H, Tanne K. Changes in the crown angulation and dental arch widths after nonextraction orthodontic treatment: model analysis of mild crowding with high canines. Open J Stomatol 2012; 2: 188-94.

18. Chan HL, Garaicoa-Pazmino C, Suarez F, Monje A, Benavides E, Oh TJ, et al. Incidence of implant buccal plate fenestration in the esthetic zone: a cone beam computed tomography study. Int J Oral Maxillofac Implants 2014; 29: 171-7.

19. Levine RA, Huynh-Ba G, Cochran DL. Soft tissue augmentation procedures for mucogingival defects in esthetic sites. Int J Oral Maxillofac Implants 2014; 29 Suppl: 155-85.

20. Demircan S, Demircan E. Dental cone beam computed tomography analyses of the anterior maxillary bone thickness for immediate implant placement. Implant Dent 2015; 24 : 664-8. 
21. Lau SL, Chow J, Li W, Chow LK. Classification of maxillary central incisors-implications for immediate implant in the esthetic zone. J Oral Maxillofac Surg 2011; 69: 142-53.

22. Buser D, Chen ST, Weber HP, Belser UC. Early implant placement following single-tooth extraction in the esthetic zone: biologic rationale and surgical procedures. Int J Periodontics Restorative Dent 2008; 28: 441-51.

23. Huynh-Ba G, Pjetursson BE, Sanz M, Cecchinato D, Ferrus J, Lindhe $\mathrm{J}$, et al. Analysis of the socket bone wall dimensions in the upper maxilla in relation to immediate implant placement. Clin Oral Implants Res 2010; 21: 37-42.

24. Kim JH, Lee JG, Han DH, Kim HJ. Morphometric analysis of the anterior region of the maxillary bone for immediate implant placement using micro-CT. Clin Anat 2011; 24: 462-8.

25. Chung SH, Park YS, Chung SH, Shon WJ. Determination of implant position for immediate implant placement in maxillary central incisors using palatal soft tissue landmarks. Int J Oral Maxillofac Implants 2014; 29: 627-33.

26. Xu D, Wang Z, Sun L, Lin Z, Wan L, Li Y, et al. Classification of the root position of the maxillary central incisors and its clinical significance in immediate implant placement. Implant
Dent 2016; 25: 520-4.

27. Jung YH, Cho BH, Hwang JJ. Analysis of the root position of the maxillary incisors in the alveolar bone using cone-beam computed tomography. Imaging Sci Dent 2017; 47: 181-7.

28. Garber DA, Belser UC. Restoration-driven implant placement with restoration-generated site development. Compend Contin Educ Dent 1995; 16: 796-804.

29. Garber DA. The esthetic dental implant: letting restoration be the guide. J Am Dent Assoc 1995; 126: 319-25.

30. Grunder U, Gracis S, Capelli M. Influence of the 3-D boneto-implant relationship on esthetics. Int J Periodontics Restorative Dent 2005; 25: 113-9.

31. Cho YB, Moon SJ, Chung CH, Kim HJ. Resorption of labial bone in maxillary anterior implant. J Adv Prosthodont 2011; 3: 85-9.

32. Araujo MG, Linder E, Lindhe J. Bio-Oss collagen in the buccal gap at immediate implants: a 6-month study in the dog. Clin Oral Implants Res 2011; 22: 1-8.

33. Mehta H, Shah S. Management of buccal gap and resorption of buccal plate in immediate implant placement: a clinical case report. J Int Oral Health 2015; 7(Suppl 1): 72-5. 